reduced to placebos. Lots of primitive treatments seem to have had real negative effects, however, as the authors later point out.

The ensuing discussion of semantics, which is again somewhat detailed but interesting nevertheless, culminates in a conceptual schema. The authors propose that the etymology of the term "placebo" should come full circle and that its definition should not be restricted to the relatively recent "inert substance" of the twentieth century. Rather, it should include active and inert substances, as well as physiological and psychological treatments. The definition of a placebo is anything that has a placebo effect. So far so good. A placebo effect is any effect that is not specific, so a placebo can be given knowingly or not. All therapies have effects that are part placebo, part non-placebo, the ratio of which the authors illustrate by the fact that predictable and specific effects increase with higher dosages. The example given is the anti-psychotic drug chlorpromazine which has a high placebo to non-placebo ratio at low, homoeopathic dosages, and a low placebo to non-placebo ratio at high dosages, indicating that the most predictable and highly specific effects occur at massive overdoses. However, the reader is left unsure as to what counts as a specific effect. Without knowledge of how a treatment works, it is difficult to know whether its effects are specific or not: perhaps doing a double-blind study would provide the answer, but then the definition may be circular. Or perhaps we could look at what effects are predicable, an approach that is implicit in the authors' discussion, but this rests on the erroneous assumption that predictability implies specificity. Massive overdoses may well lead predictably to death, but this not specific to any one disease. Mortality is a specific outcome measure, granted, but it is not the only outcome. High dosages of a drug could result in a wholesale shut-down of organs, or could at least have multiple if not fatal effects. Take aspirin for example, whose various analgesic and prophylactic effects have been well documented. Nor does specificity imply predictability. Even if we were to agree on a specific outcome, it might be difficult to predict the effects on individuals in light of population data. The definition as it stands runs the risk of being so broad as to be more or less wide open, so it is small wonder that the authors view much of prescientific therapy as placebo. The authors seem to want too much from their definition, conceding in the end that it may prove too inclusive. On the bright side, contributory factors to the placebo effect such as the natural course of the illness in question, regression to the mean, clinical bias, and methodological variables are excluded from the schema.

Quite rightly, the authors show how the introduction of scientific method to medicine and psychology reserved a respectable place for the placebo under single- and double-blind methods to evaluate therapeutics in controlled clinical trials. By controlling for the influence of patients' expectations (using single-blind studies) with additional control for observer bias (using double-blind ones), any treatment effect can be attributed to the specific pharmacological or psychological activity of the treatment in question, according to the Shapiro/Shapiro schema.

The advance of randomisation to avoid selection bias is given only brief mention. Later on in the book, the authors ask how blind is blind and provide a review of published literature plus some primary data of their own to evaluate the extent to which physicians and patients, among others, are able to guess their treatment assignment. Only tentative inferences can be drawn from the data, as the authors point out, but they still recommend the use of "matching placebos" which are identical to the experimental treatment, at least with respect to physical characteristics. There is very little in the way of discussion on the possible scientific value of "open" (non-blind) trials. By contrast, there is a good account of the relative scientific merits of placebocontrolled trials and comparative trials of two or more active agents.

There is limited analysis of the ethical issues raised by the use of placebo in practice and in research particularly. The analysis is limited both in terms of scope and depth. With regard to the former, sham operations are barely mentioned and issues such as placebo-controlled trials in Third World countries when a "proven" treatment already exists are not covered, though the authors do talk of "uncertainty" between trial arms being a moral prerequisite of trials and the unacceptability of withholding "proven" and freely available treatments from control patients. What if a country cannot afford the "proven" treatment, would it be ethical to sanction a placebo-controlled trial of a new therapy? Nor are ideas of uncertainty or, less ambiguously, equipoise taken further, which is unfortunate since the authors claim that some of the ethical constraints can be circumvented by variations in experimental design. This may indeed be so, though not necessarily in the way the authors intend. When a patient's status worsens or the patient fails to improve or develops adverse effects, the authors suggest that the clinician might remove the patient from the trial or administer an active therapy. Is this not standard procedure? In any case, the possibility that such withdrawals may compromise the scientific value of the study by introducing bias (depending on what outcome measures are used) or weaken any association, are not covered. Another difficulty, arising out of an inadequate discussion of equipoise and no mention of decision analysis, is the claim that placebo control patients may be subject to less risk than their experimental counterparts. Given genuine uncertainty (equipoise in a decision analytic framework) this may be the case, but there would be the chance of benefit for experimental patients sufficient to offset such risks. Particularly relevant here is the use of sham operations where risks may not be balanced by expected benefits. Also, the ethical implications of using "active" placebo drugs that mimic adverse effects of active treatments in an attempt to maintain the integrity of the doubleblind without promising any specific therapeutic benefit are not brought out.

SARAH J L EDWARDS

Department of Public Health and Epidemiology University of Birmingham

\section{Advance Directives or Living Wills}

\section{The Guild of Catholic Doctors, London, St Pauls, 1998, 32 pages, $£ 2.50$.}

This is a short booklet, providing advice on the use of advance directives from the perspective of the Guild of Catholic Doctors. It is the result of work of a number of members of the Guild of Catholic doctors and the Catholic Union. It is short and easy to read. The language is free from jargon and therefore accessible to a wide audience. It is a welcome contribution to the debate on the use of living wills. The authors provide their answers to 
such questions as: Can advance directives be ethically acceptable? Can treatment be ethically refused? Should advance declarations be legally binding? The booklet provides a sample Christian advance declaration for the management of serious illness.

One of the conclusions of the booklet is that many of the current advance directives are seriously objectionable on moral grounds. It is stated in support of this that: (i) the autonomous demands of patients may clash with the conscientious objections of doctors; (ii) respect for mentally incapable persons is not shown by blindly seeking to respect their past wishes but by acting in their best interests; (iii) it is morally unacceptable to refuse treatment on the basis that one's life in some anticipated condition is unacceptable and for that reason would be better ended.

The authors explicitly state that it is never morally right to withdraw assisted nutrition and hydration with the intention, purpose or aim of ending life. They go onto classify living wills into two types: the first being a specific refusal of treatment because, for example, it is too burdensome or too costly, for example, the refusal of blood products by a Jehovah's Witness and the second being the refusal of a wide range of treatments when one reaches a certain state of illness. It is suggested that the former advance refusal is ethically proper, but the latter not. It is stated that the latter is in fact to ask for death by withdrawal of treatment and is morally suicide.

The booklet is, however, slightly misleading in some respects. The authors state that the question of whether a living will is legally binding has not yet been formally tested in court. In fact a Canadian court has ruled that an applicable advance refusal of treatment is legally binding on a treating doctor and various judicial statements in courts in England and Wales have indicated that courts in this jurisdiction are likely to take the same approach.

The authors state that they agree with the view of the British Medical Association (BMA) that advance statements, although not binding on health professionals, deserve thorough consideration and respect. The BMA is, however, clear in its publication Advance Statements about Medical Treatment that where valid and applicable, advance refusals must be followed.

Whilst some people may not agree with the views and opinions expressed in the Guild of Catholic Doctors' booklet, it is an important and significant contribution to the diversity of views that have been offered on the ethical issues surrounding the use of living wills. I would recommend it to anyone concerned with the ongoing debate.

STEVEN LUTTRELL

Centre for Geriatric Medicine,

University College London Medical School

\section{Medical Harm: Historical, Conceptual and Ethical Dimensions of Iatrogenic Illness}

Virginia F Sharpe and Alan I Faden, Cambridge and New York, Methuen, 1998,280 pages, $£ 50.00$ hc, $£ 17.95$ sc.

Of the many seekers after a healthier life or of relief from suffering who rightfully expect benefit from the health care system some are more harmed than helped. This is a serious matter which the authors of this timely, readable, and informative book address seriously. ... They deal mostly with harmful medical events which happen in the course of modern management of conditions treated in acute hospitals: but the book admirably lives up to its full title.

Following a thorough, wide ranging and insightful examination of the historical and bioethical context the authors come up with the term "comiogenic harm" to best express the notion of iatrogenic illness as it occurs nowadays. They reject the pessimistic view that harm is an inevitable and inescapable concomitant of medical progress, and maintain that the corporate hubris which fosters professional hegemony, and the imperfections of cost-driven care systems are fundamental to the mala praxis that engenders avoidable illness, injury and dissatisfaction.

The book reflects the increasing public, political and economic concerns and expectations about health matters. Although the authors refer to the United States in particular their message reaches the many "Western" countries which face similar issues and tend to follow socio-economic and cultural trends set in the USA. The emergent case for an up-to-date and appropriate patient-centred fiducial contract instead of well-meaning. doctor-centred texts is clearly made.

The essential feature of such a con tract should be a binding obligation oi the health care provider to fulfil specified duty of care to the consume (patient). This demands from the pro vider personal integrity and profesy sional competence, and it gives the patient full partnership in the formula tion of the treatment plan or of the research protocol.

Topics which, unsurprisingly view of their high profile, receiv extensive coverage include: adversieu drug effects; hospital infection; unnecessary surgery, and the prematurgs introduction of unproved interven or tions. From this and other welf researched and fully documented $\mathrm{ma}=$ terial the authors conclude that much iatrogenic illness could be avoided doctors were always equipped to apple appropriate knowledge and technic skills to meet the specific needs of the individual patient. Shortcomings is these respects are seen to derive from baneful attitudes inculcated by value largely established by self-regulatin and self-seeking professional bodie that unduly influence medical educक tion and clinical training, set an $\phi$ maintain standards, and discipline miscreants. Other ways by which the interests of patients suffer include. incomplete and sometimes misleading information; restricted communication; limited participation in decision making and frustration that doctors appear to be impervious to criticist and reluctant to apply rigorous qualif. control to their output.

The authors put forward a package of measures designed to limit harm bø the promotion of good practice rather than by more stringent disciplina measures. Their demand for a moreopen and accountable medical profes sion is now widely accepted as is the need for re-negotiation of the doctore patient contract. Medical education NS undergoing one of its periodic revolugo tions with a new look that will make among other things, teaching of medical ethics mandatory and, it is to b甲 hoped, encourage both critical thinking and a more humanistic approach Clinical training and continuou medical education are now high on the agendas of professional bodies tha claim the right to ensure that the patient is safe in the hands of their members. There are helpful sections on highly relevant topics such as audit evidence based medicine, risk-benef (harm-good) analysis and the benef? cial uses of information technology. 